\title{
LA REGULACIÓN DE LA INCAPACIDAD TEMPORAL COMO FACTOR DINAMI- ZADOR DE LAS RELACIONES LABORALES: LA EXPERIENCIA ESPAÑOLA ${ }^{427}$
}

\author{
The regulation of temporary disability as a dynamic factor of labor \\ relations: the spanish experience
}

\section{Juan Carlos García Quiñones}

Profesor Contratado Doctor (Acreditado a Profesor Titular) de Derecho del Trabajo y de la Seguridad Social. Facultad de Derecho. Universidad Complutense de Madrid. E-mail: juancarlosgarciaquinones@der.ucm.es

\section{Francisca Moreno Romero}

Profesora Asociada de Derecho del Trabajo y de la Seguridad Social. Facultad de Derecho. Universidad Complutense de Madrid. E-mail: francamoro2@gmail.com

\section{RESUMEN}

La incapacidad temporal constituye una institución jurídica relevante y con una importancia singular como factor dinamizador de las relaciones laborales. Además, si se toma como base la experiencia acaecida en el ordenamiento jurídico español, su complejidad intrínseca se ha visto acrecentada con ocasión de las sucesivas reformas que se han implementado sobre la misma, con mayor virulencia si cabe durante los últimos veinte años, ausente muchas veces un planteamiento claro y permanente que garantice unos mínimos estándares de uniformidad y coherencia cada vez que ha tenido lugar un cambio legislativo de cierta significación. Un análisis que, necesariamente, debe completarse con el estudio de las técnicas de control dispuestas dentro de su propia ordenación jurídica, bien que a veces en clave de contradicción con los criterios de racionalidad, latente siempre esa apelación repetida a la construcción de un nuevo modelo de gestión y control. Del mismo modo que resulta necesario reflexionar mínimamente sobre la necesaria interacción entre la incapacidad temporal y el contrato de trabajo. Aspectos todos que pueden servir de base para una aproximación científica a la regulación de la incapacidad temporal como factor dinamizador de las relaciones laborales, a partir de la experiencia que ofrece

${ }^{427}$ La presente Comunicación se inserta en las actividades del Proyecto de Investigación "La Incapacidad Temporal en el trabajo de hombres y mujeres" (Subprograma de Proyectos de Investigación Fundamenta no orientada, convocatoria 2011 (DER2011-29448-C03-01). 
el ordenamiento español. Y elementos que deben analizarse también, en clave de actualidad, a la luz de lo dispuesto en la última manifestación legal, el Real Decreto $625 / 2014$, de 18 de julio, por el que se regulan determinados aspectos de la gestión y control de los procesos por incapacidad temporal en los primeros trescientos sesenta y cinco días de su duración.

Palabras clave: Incapacidad temporal. Técnicas de control. Contrato de trabaJO.

\section{Abstract}

Temporarily unable for work is a relevant legal institution and a factor singular importance in the dynamics of labor relations. Also, if you take on the experience occurred in the Spanish legal system, its inherent complexity has been increased during the successive reforms that have been implemented on same, more virulent if possible during the last twenty years, many missing sometimes a clear and ongoing approach to ensure minimum standards of uniformity and consistency every time has been a major legislative change. An analysis must necessarily be supplemented by the study of control techniques provided within its own legal order, albeit sometimes in contradiction with the key criteria of rationality. In addition to appealing to the construction of a new model of management and control. And conclude with a reflection on the necessary interaction between temporary disability and the employment contract. All aspects that can be the basis for the scientific approach to the regulation of temporary disability as a dynamic factor in industrial relations from the experience offered by the Spanish system.

KeYWords: TEMPORARILY UNABLE FOR WORK. CONTROL TECHNIQUES. EMPLOYMENT CONTRACT.

SUMARIO: 1. Retrospectiva histórica del tratamiento legislativo dado a la incapacidad temporal. 2. Problemática inherente a la regulación de la incapacidad temporal. 3. Técnicas de control versus racionalidad en la ordenación jurídica: la construcción de un nuevo modelo de gestión y control. 4. Una última manifestación legal relevante: El Real Decreto 625/2014, de 18 de julio, por el que se regulan determinados aspectos de la gestión y control de los procesos por incapacidad temporal en los primeros trescientos sesenta y cinco días de su duración. 4.1. Un tópico en la incapacidad temporal: gasto y fraude como justificativos del control. 4.2. La ubicación y los espacios del RD 625/2014 de 18 de Julio. 4.3. La fragmentación temporal de los procesos y la hegemonía de los protocolos. 4.3.1. Instrumentos de control sobre la actuación de los facultativos y centralización de la información. 4.3.2. La fragmentación temporal de los procesos como mecanismo control-presión. Conclusión. Bibliografía 


\section{INTRODUCCIÓN}

La incapacidad temporal constituye una institución jurídica relevante y con una importancia singular como factor dinamizador de las relaciones laborales. Además, si se toma como base la experiencia acaecida en el ordenamiento jurídico español, su complejidad intrínseca se ha visto acrecentada con ocasión de las sucesivas reformas que se han implementado sobre la misma, con mayor virulencia si cabe durante los últimos veinte años, ausente muchas veces un planteamiento claro y permanente que garantice unos mínimos estándares de uniformidad y coherencia cada vez que ha tenido lugar un cambio legislativo de cierta significación. Un análisis que, necesariamente, debe completarse con el estudio de las técnicas de control dispuestas dentro de su propia ordenación jurídica, bien que a veces en clave de contradicción con los criterios de racionalidad, latente siempre esa apelación repetida a la construcción de un nuevo modelo de gestión y control.

Del mismo modo que resulta necesario reflexionar mínimamente sobre la necesaria interacción entre la incapacidad temporal y el contrato de trabajo. Aspectos todos que pueden servir de base para una aproximación científica a la regulación de la incapacidad temporal como factor dinamizador de las relaciones laborales, a partir de la experiencia que ofrece el ordenamiento español. Y elementos que deben analizarse también, en clave de actualidad, a la luz de lo dispuesto en la última manifestación legal, el Real Decreto 625/2014, de 18 de julio, por el que se regulan determinados aspectos de la gestión y control de los procesos por incapacidad temporal en los primeros trescientos sesenta y cinco días de su duración.

En definitiva, aspectos todos cuyo análisis en clave integradora puede servir de base para esa aproximación científica a la regulación de la incapacidad temporal como factor dinamizador de las relaciones laborales, desde la experiencia que ofrece el ordenamiento español. Y examen donde no puede faltar una referencia concreta a la última manifestación legal de relevancia, por alusión al Real Decreto $625 / 2014$, de 18 de julio, por el que se regulan determinados aspectos de la gestión y control de los procesos por incapacidad temporal en los primeros trescientos sesenta y cinco días de su duración

\section{RETROSPECTIVA HISTÓRICA DEL TRATAMIENTO LEGISLATIVO DADO A LA INCA- PACIDAD TEMPORAL}

Las viejas y nunca pacíficas contingencias de incapacidad laboral transitoria e invalidez provisional, fueron reformadas por la Ley 42/1994, de 27 de diciembre, de Medidas fiscales, administrativas y del orden social, que procedió a la redenominación de la contingencia -incapacidad temporal- y a reformar parcialmente su régimen jurídico, suprimiendo formalmente la invalidez provisional. A la referida reforma habría que añadir otras anteriores (por ejemplo, la efectuada en materia de cuantía de la prestación y responsables del pago por la Ley 42/1992, 
de 24 de noviembre, cuya constitucionalidad fue declarada por STCO 34/1994, de 10 de febrero) y posteriores ,por ejemplo, la efectuada en materia de extinción de la prestación económica por las Leyes 66/1997, de 30 de diciembre y 24/2001, de 27 de diciembre, así como por el Real Decreto-Ley 6/2000, de 23 de junio. Junto a ellas, las más recientes incorporadas por la Ley 30/2005, de 29 de diciembre de Presupuestos Generales del Estado para el año 2006, la Ley 40/2007, de 4 de diciembre, de Medidas en Materia de Seguridad Social que rectifica parcialmente las reformas del 2006, las recogidas en la disposición final tercera de la Ley 26/2009, de 24 de diciembre, de Presupuestos Generales del Estado para el año 2010 y, finalmente, las incorporadas a la Ley 35/2010, de 17 de septiembre, de medidas urgentes para la reforma del mercado de trabajo.

Las reformas referidas, aunque importantes, no son las únicas, todavía habría que añadir otras múltiples reformas directas de alcance reglamentario y otras indirectas que proceden de la reforma de otras contingencias, de materias e instituciones que son comunes al Sistema, o finalmente, de normas procedentes de otras parcelas del ordenamiento, singularmente del Estatuto de los Trabajadores. De igual modo que merece significarse también cómo, en el conjunto de la normativa referida, la "ausencia del empresario es absoluta", a pesar de soportar una parte importante del gasto directo e indirecto. Normativa reguladora de las facultades del empresario que sigue invariable desde la incorporación del artículo 20.4 en la primera versión del Estatuto de los Trabajadores.

Por lo demás, toda la complejidad normativa referida se ha producido manteniéndose la ordenación reglamentaria procedente del desarrollo de la Ley de Seguridad Social de 1966 (singularmente el Decreto 3158/1966, de 23 de diciembre y la Orden de 13 de octubre de 1967, que han encajado múltiples reformas) que convive con todo un elenco de normas reglamentarias posteriores de distinta procedencia y alcance (desde el Decreto 1646/1972, de 23 de junio, pasando por el Real Decreto 53/1980, de 11 de enero, hasta los más recientes sobre gestión y control, el Real Decreto 575/1997, de 18 de abril, modificado por el Real Decreto 1117/1998, de 5 de junio y el Real Decreto 430/2009, de 1 de septiembre, de desarrollo reglamentario de la Ley 40/2007), incluida la aplicación de las modernas tecnologías para la presentación en soporte informático de los partes médicos (O Tas/399/2004, de 12 de febrero). Sobre esta anacrónica y compleja estructura normativa, han impactado reformas procedentes de la incapacidad permanente, la maternidad, el riesgo durante el embarazo, la jubilación parcial, el desempleo..., lo que dificulta su comprensión. Finalmente, algunas de las reformas producidas, singularmente las que han afectado a la Ley General de Seguridad Social, no han tenido desarrollo reglamentario, dejando vacíos normativos que se resuelven por criterios internos de las entidades gestoras o por decisiones jurisprudenciales no siempre afortunadas. 


\section{Problemática inherente a la Regulación de la INCAPACIDAd temporal}

La evolución desordenada, en cierto modo asistemática, de la que ha sido objeto la incapacidad temporal explica también el interés mostrado por la doctrina científica sobre determinadas materias centrales relacionadas con la misma, como sucede de manera específica con las vacaciones y la incapacidad temporal en la Administración General del Estado ${ }^{428}$; o desde una proyección más amplia, con el estudio de la incapacidad temporal de los funcionarios públicos ${ }^{429}$; el derecho al disfrute efectivo de las vacaciones, a partir de las referencias básicas que suponen la jurisprudencia comunitaria y la regulación estatutaria ${ }^{430}$; apelación a las vacaciones desde variantes distintas que dan cuenta de la compleja relación existente entre la prestación por incapacidad temporal y el derecho de vacaciones, considerando también los nuevos criterios judiciales y legales ${ }^{431}$; la incapacidad temporal como causa de despido ${ }^{432}$; y, en directa conexión con lo anterior, con el referente de la tutela del trabajador enfermo dentro del Estatuto de los Trabajadores ${ }^{433}$; desde el examen omnicomprensivo de la protección de la incapacidad temporal en el régimen general de la Seguridad Social ${ }^{434}$; también a la luz de la evolución de la normativa sobre

${ }^{428}$ ARRIBAS LÓPEZ, E., El tan traído y llevado tema de las vacaciones y la incapacidad temporal en la Administración General del Estado, Actualidad Administrativa, núm. 4, 2014, Págs. 403 y ss.

${ }^{429}$ ARETA MARTÍNEZ, Ma ${ }^{\text {. }}$, La incapacidad temporal de los funcionarios públicos, AA. VV.: Cuestiones en torno a la incapacidad temporal de los funcionarios públicos, Cinca, Madrid, 2011, págs.. 198 y ss.

${ }^{430}$ En este sentido, GORELLI HERNÁNDEZ, J., El derecho al disfrute efectivo de las vacaciones: de la jurisprudencia comunitaria a la regulación estatutaria, Relaciones Laborales, núm. 1, 2014, págs. 1 y ss.; LÓPEZ INSUA, B. M., La incapacidad temporal en el Sistema de la Seguridad Social, Comares, Granada, 2014; Vacaciones e Incapacidad Temporal: perspectiva comunitaria y española, Aranzadi Social Revista Doctrinal, núm. 10, 2013, págs.. 243 y ss.; RODRÍGUEZ-PIÑERO, M., Derecho a vacaciones y baja del trabajador en la reciente jurisprudencia del TJCE, Relaciones Laborales, núms. 13/14, 2012, págs. 1 y ss.

${ }^{431}$ GALA DURÁN, C., La compleja relación entre la prestación por incapacidad temporal y el derecho a vacaciones: nuevos criterios judiciales y legales, La administración pública, núm. 10, 2012, págs. 916 y ss.

${ }^{432}$ ARENAS VIRUEZ, M., La incapacidad temporal como causa de despido: nulidad o improcedencia, Relaciones Laborales, núm. 1, 2011, págs. 21 y ss.;AZAGRA SOLANO, M., Reflexiones sobre el despido del trabajador enfermo, Revista Aranzadi Doctrinal, núm. 4, 2011, págs. 57 y ss.; SAN MARTÍN MAZZUCONI, C., El despido del trabajador en situación de incapacidad temporal, AA. VV.: Cuestiones en torno a la Incapacidad Temporal (Dir. SEMPERE NAVARRO, A. V.), Cinca, Madrid, 2011, págs. 49 y ss.

${ }^{433}$ CARRIZOSA PRIETO, E., La tutela del trabajador enfermo en el Estatuto de los Trabajadores, Revista Española de Derecho del Trabajo, núm. 157, 2013, págs. 135 y ss.

${ }^{434}$ FERNÁNDEZ PRATS, C., La protección de la incapacidad temporal en el régimen ge- 
la incapacidad para el trabajo, siempre con la referencia del ordenamiento jurídico español ${ }^{435}$; o, de manera más acotada en el tiempo, considerando la incidencia de la reforma laboral de 2012 en el sistema público de la Seguridad Social ${ }^{436}$; desde la interdependencia entre la incapacidad temporal y el presente y futuro de las mutuas de accidentes de trabajo y enfermedad profesional ${ }^{437}$; a partir de una triple problemática proyectada sobre la incapacidad temporal, con una dimensión legal, jurisprudencial y propiamente médica ${ }^{438}$; analizando la evolución de la jurisprudencia del Tribunal Supremo respecto de la compatibilidad entre el trabajo y la pensión de incapaci$\operatorname{dad}^{439}$; o por la proyección de la incapacidad temporal vinculada con determinados colectivos específicos de trabajadores, como sucede significativamente respecto de los trabajadores autónomos ${ }^{440}$; o por su juego en relación con determinadas modalidades de contratos, en alusión al trabajador a tiempo parcial y los trabajadores fijos discontinuos ${ }^{441}$.

Problemática acrecentada cuando se constata que, tanto en la vieja normativa como en la nueva, el impacto y las relaciones reciprocas entre la incapacidad temporal y el contrato de trabajo, son permanentemente ignorados, lo que provoca importantes problemas aplicativos. La importancia del tema y la interrelación de

neral de la Seguridad Social, Tirant lo Blanch, Valencia, 2011; LÓPEZ INSUA, B. M., La incapacidad temporal en el Sistema de la Seguridad Social, Comares, Granada, 2014; Vacaciones e Incapacidad Temporal: perspectiva comunitaria y española, Aranzadi Social Revista Doctrinal, núm. 10, 2013, págs. 45 y ss.

${ }^{435}$ RODRÍGUEZ ÁLVAREZ, V., Evolución de la normativa sobre la incapacidad para el trabajo en el ordenamiento jurídico español, Revista del Ministerio de Trabajo e Inmigración, núm. 94, 2011, págs. 173 y ss.

${ }^{436}$ BLASCO LAHOZ, J. F., La incidencia de la reforma laboral en el sistema público de la Seguridad Social, AA. VV.: Tres años de cambio laboral, Lex Nova, Valladolid, 2013; Págs. 20121 y ss.

${ }^{437}$ BLÁZQUEZ AGUDO, E. Ma ., Presente y futuro de las mutuas de accidentes de trabajo y enfermedad profesional, Aranzadi Social Revista Doctrinal, núm. 2, 2012, págs.. 373 y ss.

${ }^{438}$ BRUNA REVERTER, J., La incapacidad laboral: problemática legal, jurisprudencial y médica, Comares, Granada, 2012, págs. 1 y ss.

${ }^{439}$ FERNÁNDEZ LOMANA, M., Compatibilidad trabajo-pensión de incapacidad: evolución de la jurisprudencia del Tribunal Supremo, Actualidad Laboral, núm. 2, 2013, págs. 181 y ss.

${ }^{440}$ CASTRO CONTE, M., Evolución de la Seguridad Social de los trabajadores autónomos o por cuenta propia, AA. VV.: Cuestiones en torno a la Incapacidad Temporal (Dir. SEMPERE NAVARRO, A. V.), Cinca, Madrid, 2011; GARCÍA QUIÑONES, J. C., Ampliación de la prestación por incapacidad temporal para los trabajadores por cuenta propia, Documentación Laboral, núm. 70, 2004, págs. 59 y ss.

${ }^{441}$ MEDINA MAILHO, R., Trabajo a tiempo parcial y fijos discontinuos, AA. VV.: Cuestiones en torno a la Incapacidad Temporal (Dir. SEMPERE NAVARRO, A. V.), Cinca, Madrid, 2011, págs. 151 y ss. 
ambos institutos es clara, ya que el efecto incapacitante temporal que constituye el elemento de protección es, a su vez, la causa que motiva la suspensión del contrato de trabajo. De ello deriva que las reformas que actúan sobre la incapacidad temporal (prorrogas, suspensión cautelar, procedimiento de impugnación del alta médica, extinción...) provocan sus efectos sobre el contrato de trabajo.

\section{TÉCNICAS DE CONTROL VERSUS RACIONALIDAd EN LA ORDENACIÓN JURÍDI- CA: LA CONSTRUCCIÓN DE UN NUEVO MODELO DE GESTIÓN Y CONTROL}

El análisis de la incapacidad temporal debe completarse, necesariamente, con el estudio de las técnicas de control dispuestas dentro de su propia ordenación jurídica, bien que a veces en clave de contradicción con los criterios de racionalidad. Materia que constituye, de igual modo, un referente continuo de atención por parte de la doctrina científica, cuando analiza la conexión de la incapacidad temporal con el control público del absentismo laboral ${ }^{442}$; con la atención puesta en el control médico de la incapacidad temporal desde la reordenación de las competencias de las entidades que intervienen en la prestación citada, a partir de la regulación que establece el artículo 128 de la Ley General de Seguridad Social ${ }^{443}$; constatando la relevancia del control de las bajas médicas como objetivo permanente de las sucesivas reformas acaecidas en materia de incapacidad temporal ${ }^{444}$; con atención sobre el control y la gestión de la incapacidad temporal a partir de un análisis crítico del marco jurídico positivo vigente, conscientes en cualquier caso del equilibrio complejo que exige la preservación de elementos necesarios a considerar como son la eficiencia y el garantismo ${ }^{445}$; atendiendo a la incidencia que reviste el control de la incapacidad temporal sobre la contención del gasto público y el aumento de la productividad

${ }^{442}$ PURCALLA BONILLA, M. A., Incapacidad temporal y control del absentismo, AA. VV.: Régimen jurídico y gestión racional del tiempo en la empresa (Dirs. ROMERO BURILLO, A. Ma y ARGÜELLES BLANCO, A. R.), Aranzadi, Cizur Menor, 2013, págs. 227 y ss.

${ }^{443}$ CASTILLA CASTILLA, Á., Control médico de la Incapacidad Temporal: la reordenación de las competencias de las entidades que intervienen en la prestación de la Incapacidad Temporal: artículo 128 de la LGSS, Revista de Información Laboral, núm. 3, 2012, págs. 17 y ss. ${ }^{444}$ GONZÁLEZ ORTEGA, S., El control de las bajas médicas como objetivo permanente de las reformas de la incapacidad temporal (I) (El control durante la primera fase de la incapacidad temporal, Relaciones Laborales, núm. 12, 2011, págs. 11 y ss.; El control de las bajas médicas como objetivo permanente de las reformas de la incapacidad temporal (y II) (Prórrogas y recaídas. El tránsito hacia la incapacidad permanente), Relaciones Laborales, núm. 13, 2011, págs. 19 y ss.

${ }^{445}$ OLARTE ENCABO, S., Control y gestión de la incapacidad temporal: análisis crítico del marco jurídico-positivo vigente, el complejo equilibrio entre eficiencia y garantismo, Aranzadi Social Revista Doctrinal, núm. 20, 2011, págs.. 63 y ss. 
empresarial ${ }^{446}$; o para corroborar la opción preferencial por el control público frente al poder de dirección del empresario, dentro de los mecanismos de control de la incapacidad temporal ${ }^{447}$. Elementos todos que están en la base de una llamada, repetida y recurrente, a la construcción de un nuevo modelo de gestión y control.

Como es notorio, la problemática que afecta a la ordenación, gestión y control de la incapacidad temporal, es compleja en sí misma y exige clarificar los condicionamientos sociales y jurídicos sobre los que opera, con la finalidad de fijar el rumbo de las posibles reformas futuras o, al menos, evidenciar algunos de los elementos que proporcionan la complejidad anunciada. Con todo, se trata de una cuestión nuclear en la ordenación de la incapacidad temporal, que afecta a todos los intervinientes, a saber: Servicio Público de Salud, Mutua de accidentes de trabajo y enfermedades profesionales, Instituto Nacional de la Seguridad Social, Instituto Social de la Marina, empresario y trabajador, así como, en ocasiones, al Servicio Público de Empleo Estatal. Singularidad ésta que al ser exclusiva de la incapacidad temporal, anuncia por sí misma su complejidad.

En definitiva, el conjunto de cuestiones enumeradas evidencia a las claras cómo la incapacidad temporal, al igual que otras contingencias de la Seguridad Social, tiene una ordenación sumamente compleja, caótica y desordenada, que dificulta su comprensión y aplicación, situándonos en un espacio de inseguridad jurídica difícilmente justificable.

Situación que no se ha visto favorecida por el hecho de que en los últimos veinte años, desde 1992, la incapacidad temporal ha sido el centro de atención del legislador, realizando reformas continuas -y no siempre debidamente maduradas- con la finalidad de construir un nuevo modelo de gestión y control, que permita recomponer el papel que deben asumir las entidades intervinientes en el proceso, reconducir su utilización -no siempre ortodoxa- y situar el gasto en parámetros de racionalidad. Un entramado normativo que ha tenido como objetivo principal la creación de un modelo de gestión y control bajo la pretensión de centralizar los espacios de competencia. Y materia en la que incide, de manera directa, la aprobación por el Gobierno del Real Decreto 625/2014, de 18 de julio, por el que se regulan determinados aspectos de la gestión y control de los procesos por incapacidad temporal en los primeros trescientos sesenta y cinco días de su duración, bajo esa pretensión declarada de afianzar el modelo en construcción y singularmente incorporar a la gestión los procesos telemáticos, con la finalidad de que todos los "agentes" intervinientes operen y

${ }^{446}$ RODRÍGUEZ ESCANCIANO, S., El control de la incapacidad temporal: su incidencia sobre la contención del gasto público y el aumento de la productividad empresarial, Temas Laborales, núm. 118, 2013, págs. 113 y ss.

447 TORTUERO PLAZA, J. L., La incapacidad temporal, contingencia y situaciones protegidas: un análisis teórico, Tribuna Social, núms. 44/45, 1994; El control público de las Mutuas en materia de incapacidad temporal, AA. VV.: Accidentes de trabajo y Mutuas, La Ley, Madrid, 2008, págs. 217 y ss. 
dispongan de toda la información clínica del trabajador. Texto legal que analizamos, de manera específica, en el epígrafe que sigue a continuación.

\section{Una Última manifestación legal ReleVante: El Real Decreto 625/2014, DE 18 DE JULIO, POR EL QUE SE REGULAN DETERMINADOS ASPECTOS DE LA GES- TIÓN Y CONTROL DE LOS PROCESOS POR INCAPACIDAD TEMPORAL EN LOS PRIME- ROS TRESCIENTOS SESENTA Y CINCO DÍAS DE SU DURACIÓN}

\subsection{UN TÓPICO EN LA INCAPACIDAD TEMPORAL: GASTO Y FRAUDE COMO JUSTIFI- CATIVOS DEL CONTROL}

Tradicionalmente, el incremento del gasto en incapacidad temporal ha propiciado que las principales reformas de los últimos años se hayan centrado casi en exclusividad sobre la ordenación de los mecanismos de control, bajo la hipótesis de que un mayor control conducirá a una reducción del gasto. No obstante, las reformas no se plantearon la problemática existente en forma sosegada y reflexiva, sino más bien conducidas por la obsesión razonable del gasto y la idea de que el fin justifica cualquier medio. En este orden, el aluvión normativo producido entre el año 1997 y el año 2014, ha buscado la reordenación de los mecanismos de control con la finalidad de alcanzar el resultado económico deseado.

Junto a las razones económicas y/o vinculado a ellas, el espacio competencial ha constituido el eje direccional de las reformas, sin que se haya logrado el equilibrio necesario. En esta línea, todas las reformas, con mayor o menor fortuna, se han dirigido a convertir al Instituto Nacional de la Seguridad Social -y en su espacio competencial al Instituto Social de la Marina- en parte, juez, fiscal instructor... con una centralización de competencias que, como ha afirmado la doctrina ${ }^{448}$, plantean dudas razonables en la construcción de un modelo de gestión equilibrado.

En un diálogo entre competencias y nivel de gasto, podríamos decir que las últimas reformas ha primado el elemento competencial ${ }^{449}$. La crisis económica se ha encargado por sÍ sola de reducir el gasto, tanto por la pérdida de afiliados, como por la reducción de salarios y cotizaciones -factores externos que condicionan el gasto en incapacidad temporal- e, incluso, por el temor razonable del trabajador a perder el empleo, temor que ha primado sobre su derecho a la salud.

Es frecuente imputar a los trabajadores el uso incorrecto de la incapacidad

448 AA. VV.: Cuestiones en torno a la Incapacidad Temporal (Dir. SEMPERE NAVARRO, A. V.), Cinca, Madrid, 2011; TORTUERO PLAZA, J. L El control público de las Mutuas en materia de incapacidad temporal, AA. VV.: Accidentes de trabajo y Mutuas, La Ley, Madrid, 2008, págs. 137 y ss.

449 PANIZO ROBLES, J. A., Un nuevo paso en el control de la prestación de la seguridad social por incapacidad temporal: el RD 1430/2009, de 4 de diciembre, Revista de Trabajo y Seguridad Social, CEF, núm. 320, 2009, pág. 3. 
temporal, en el sentido de que es utilizada o mantenida temporalmente, al margen del efecto incapacitante que la caracteriza. Igualmente lo es, imputar a los facultativos del servicio público de salud de falta de rigor en la apertura y mantenimiento de los procesos de incapacidad temporal. Las imputaciones no son nuevas, más bien son consustanciales a la contingencia misma y de ellas hemos hecho un tópico que incluso constituye guía y razón de las reformas legislativas. Es cierto que no sólo debemos valorar el importante impacto económico que tiene sobre el sistema público de protección social y sobre las empresas, sino también los importantes impactos colaterales. Así, el absentismo causado por la incapacidad temporal, tiene importantes repercusiones que afectan a la productividad y competitividad de las empresas, a los espacios organizativos, a la gestión de personal... ${ }^{450}$. Las soluciones sobrepasan los espacios de control, sobre todo cuando se rompe el necesario equilibrio entre derecho a la salud, protección y control. Múltiples son las valoraciones y análisis realizados por la doctrina, al plantearse la incapacidad, como un "refugio protegido", como un instrumento del "compensar las irregularidades del mercado laboral", su "impacto de determinadas actividades económicas", sobre la "función social impropia que cumple". Sin embargo, el legislador lejos de profundizar en estos espacios para buscar soluciones equilibradas, ha optado por la creación de múltiples mecanismos de control ejercitables desde todas las instituciones intervinientes, los inspectores médicos del Instituto Nacional de la Seguridad Social, los facultativos y la Inspección del servicio público de salud, los facultativos de las mutuas y el empresario. Controles y actuaciones seguidos -en ocasiones- de un abanico de procedimientos de impugnación, revisión, determinación de la contingencia, etc. Todo ello completado con un sinfín de convenios de colaboración entre todas las entidades intervinientes, con las correspondientes dotaciones económicas. Las dudas sobre el equilibrio final son más que razonables.

\subsection{La ubiCaCión y LOS ESPACIOS DEL RD 625/2014 de 18 DE JULIO}

Las normas sobre gestión y control de la incapacidad temporal contempladas en el Real Decreto 575/1997, de 18 de abril y sus normas de desarrollo habían quedado obsoletas. Desde aquellos años las reformas operadas en la Ley General de la Seguridad Social han sido múltiples y han ido poco a poco cambiando el modelo de gestión de la incapacidad temporal. Era necesario por tanto proceder a su actualización e incluso a la armonización de toda la normativa reglamentaria referida a la contingencia.

En este espacio se sitúa el RD 625/2014, que deroga el viejo RD 575/1997. La sustitución significa actualización /modernización con respecto a las reformas de la LGSS, pero como veremos no significa armonización de la normas reglamen-

${ }^{450}$ MERCADER UGUINA, J. El control de la incapacidad temporal (historia de una sospecha), Revista de Relaciones Laborales, Tomo I, 2002, pág. 413. 
tarias. Lamentablemente se ha perdido la oportunidad de afrontar la ordenación de todas las materias vinculadas a la gestión y control, tanto durante los primeros trescientos sesenta y cinco días - periodo éste en el que se sitúa el RD- como de los tiempos posteriores hasta alcanzar su duración máxima de setecientos treinta días.

Las materias objeto de reforma son múltiples y de distinto alcance. A modo de resumen afectan a las siguientes materias:

- La nueva ordenación es aplicable a los primeros 365 día, cualquiera que sea la contingencia determinante - accidente de trabajo, enfermedad profesional, enfermedad común y accidente no laboral- y cualquiera que sea en régimen - general o especiales-, reflejando su vocación universal.

- Deja fuera como suele ser habitual a los denominados los regímenes especiales de las Fuerzas Armadas, de los Funcionarios Civiles de la Administración del Estado y del personal al servicio de la Administración de Justicia.

- Se moderniza la gestión formal (partes de baja, confirmación y alta) diferenciando los procesos según su duración estimada - inferior a cinco día, entre 5 y 30 días naturales; entre 31 y 60 y entre 61 y más días- en base a protocolos técnico-sanitarios, con identificación de los sujetos responsables en función de la contingencia y de los contenidos de los nuevos partes. - Se apuesta por la gestión telemática en todas las fases y respecto de todos los intervinientes.

- Se reconoce y facilita el acceso a la información clínica del trabajador incapacitado en una ordenación extendida a "los trabajadores del sistema de seguridad social, que posiblemente vulnera por su amplitud los límites legales establecidos en la Ley Orgánica 1/1982, de 5 de mayo, sobre protección civil del derecho al honor, a la intimidad personal y familiar y a la propia imagen, dictada en desarrollo del artículo 18.1 CE.

- Ordena el procedimiento para las propuestas de alta médica formuladas por las mutuas en los procesos derivados de contingencias comunes.

- Ordena, en desarrollo del artículo 132.2 de la LGSS, los requerimientos para reconocimientos médicos de control y la suspensión cautelar de la prestación ante la falta de comparecencia del trabajador.

- Incorpora un nuevo artículo 6 al RD 1430/2009, donde se regula el procedimiento administrativo de determinación de la contingencia causante en los procesos de incapacidad temporal.

- Igualmente, incorpora un nuevo artículo 7 al RD 1430/2009, donde se regula la prolongación de efectos de la incapacidad temporal y agotamiento de la misma. 


\subsection{LA FRAGMENTACIÓN TEMPORAL DE LOS PROCESOS Y LA HEGEMONÍA DE LOS PROTOCOLOS}

\subsubsection{INSTRUMENTOS DE CONTROL SOBRE LA ACTUACIÓN DE LOS FACULTATIVOS Y CENTRALIZACIÓN DE LA INFORMACIÓN}

La nueva ordenación realizada por el RD 625/2014 de los elementos formales vinculados al nacimiento, duración y extinción de la incapacidad temporal - baja médica, confirmación y alta-, son estructurados de forma que en sí mismos constituyen mecanismos de control que pretenden actuar contra la utilización inadecuada de la contingencia, tanto en lo que refiere a su nacimiento, como en su duración. Los instrumentos son múltiples - imposición de protocolos clínicos, fragmentación temporal de los procesos, códigos de diagnostico, código nacional de ocupación, informes complementarios...- y pretenden controlar la acción médica y centralizar la información, cualquiera que sea la entidad gestora, a través de medios telemáticos en el Instituto Nacional de la Seguridad Social.

El parte médico de baja es el acto constitutivo de la incapacidad temporal que, por un lado provoca la suspensión del contrato de trabajo y, por otro, determina el nacimiento de la incapacidad temporal. De esta forma, el parte médico de baja irá precedido de un reconocimiento médico del trabajador que permita la determinación objetiva de la incapacidad para el trabajo habitual, a cuyo efecto el médico requerirá al trabajador los datos necesarios que contribuyan tanto a precisar la patología objeto de diagnóstico, como su posible incapacidad para realizar su trabajo. Lo que impone el precepto, según el artículo 2 del RD, es que el facultativo tenga toda la información, tanto en los aspectos clínicos, como en los vinculados a la actividad laboral del trabajador - tipo de actividad, contenido obligacional, forma de prestar la actividad, condicionantes...-, con la finalidad de poder realizar la correspondiente evaluación que determine o no el efecto incapacitante de la patología.

Con el fin de que las actuaciones médicas cuenten con el mayor respaldo técnico se pondrá a disposición de los médicos a los que competan dichas actuaciones, tablas de duración óptima tipificadas por los distintos procesos patológicos susceptibles de generar incapacidades, así como tablas sobre el grado de incidencia de aquellos procesos en las distintas actividades laborales.

Los partes médicos de incapacidad temporal se confeccionarán con arreglo a un modelo que permita su gestión informatizada, en el que figurará un código identificativo del centro de salud emisor de aquellos.

Desde el momento inicial, el Instituto Nacional de la Seguridad Social centraliza toda la información. Así, el servicio público de salud o la mutua, según cuál sea la entidad facultada para emitir el parte de baja, remitirá por vía telemática- al INSS- de manera inmediata y en todo caso, en el primer día hábil : los datos personales del trabajador y, además, los datos obligatorios del parte de baja relativos a la 
fecha de la baja, a la contingencia causante, al código de diagnóstico, al código nacional de ocupación del trabajador, a la duración estimada del proceso y, en su caso, la aclaración de que el proceso es recaída de uno anterior, así como, en este caso, la fecha de la baja del proceso que lo origina. Asimismo, hará constar la fecha en que se realizará el siguiente reconocimiento médico.

\subsubsection{La fragmentación temporal de los procesos como mecanismo control -presión}

Otro de los mecanismos de control novedosos se centra en la fragmentación de los procesos de incapacidad en función de su posible duración, lógicamente según los protocolos aplicables. A pesar del revestimiento de buenas intenciones y justificaciones tecnológicas contenidas en la Exposición de Motivos se hace patente expresamente -se cuela en el discurso- la clara intencionalidad del "control".

Como veremos, tras la apariencia de facilitar la expedición y tramitación de los partes médicos, se ejercita un drástico mecanismo de control y de presión sobre el facultativo y sobre el trabajador, al predeterminarse la fecha de alta médica en cada proceso. La propia Exposición de Motivos del RD, ofrece como justificación que “... es importante modificar el modelo actual dando la oportunidad al médico de atención primaria de que determine cuándo tiene que hacer un seguimiento de la enfermedad de su paciente sin condicionarlo, como actualmente, a que semanalmente deba expedir un parte médico de confirmación de la baja".

Los partes de baja y de confirmación de la baja se extenderán en función del periodo de duración que estime el médico que los emite. A estos efectos se establecen cuatro grupos de procesos:

a) En los procesos de duración estimada inferior a cinco días naturales, el facultativo del servicio público de salud, o de la mutua, emitirá el parte de baja y el parte de alta en el mismo acto médico.

El facultativo, en función de cuando prevea que el trabajador va a recuperar su capacidad laboral -los protocolos-, consignará en el parte la fecha del alta, que podrá ser la misma que la de la baja o cualquiera de los tres días naturales siguientes a ésta.

No obstante el trabajador podrá solicitar que se le realice un reconocimiento médico el día que se haya fijado como fecha de alta y el facultativo podrá emitir el parte de confirmación de la baja, si considerase que el trabajador no ha recuperado su capacidad laboral.

b) En los procesos de duración estimada de entre 5 y 30 días naturales, el facultativo del servicio público de salud, o de la mutua, emitirá el parte de baja consignando en el mismo la fecha de la revisión médica prevista que, en ningún caso, excederá en más de siete días naturales a la fecha de baja 
inicial. En la fecha de revisión se extenderá el parte de alta o, en caso de permanecer la incapacidad, el parte de confirmación de la baja. Después de este primer parte de confirmación, los sucesivos, cuando sean necesarios, no podrán emitirse con una diferencia de más de catorce días naturales entre sí.

c) En los procesos de duración estimada de entre 31 y 60 días naturales, el facultativo del servicio público de salud, o de la mutua, emitirá el parte de baja consignando en el mismo la fecha de la revisión médica prevista que, en ningún caso, excederá en más de siete días naturales a la fecha de baja inicial, expidiéndose entonces el parte de alta o, en su caso, el correspondiente parte de confirmación de la baja. Después de este primer parte de confirmación, los sucesivos, cuando sean necesarios, no podrán emitirse con una diferencia de más de veintiocho días naturales entre sí.

d) En los procesos de duración estimada de 61 o más días naturales, el facultativo del servicio público de salud, o de la mutua, emitirá el parte de baja en el que fijará la fecha de la revisión médica prevista, la cual en ningún caso excederá en más de catorce días naturales a la fecha de baja inicial, expidiéndose entonces el parte de alta o, en su caso, el correspondiente parte de confirmación de la baja. Después de este primer parte de confirmación, los sucesivos, cuando sean necesarios, no podrán emitirse con una diferencia de más de treinta y cinco días naturales entre sí.

Siempre que se produzca una modificación o actualización del diagnóstico, se emitirá un parte de confirmación que recogerá la duración estimada por el médico que lo emite. Los siguientes partes de confirmación se expedirán en función de la nueva duración estimada.

El elemento de presión-control consistente en la predeterminación temporal del parte de alta médica y, por tanto, de la extinción de la incapacidad temporal tiene posiblemente un efecto adicional. Cuando el alta predeterminada no se consolide, bien por la modificación del diagnóstico, bien por la evolución del propio proceso, actuará como señal de alarma para la actuación puntual de los inspectores médicos del Instituto Nacional de la Seguridad Social, en la medida en que tienen competencias para expedir el alta médica. Actuación que, como hemos visto, viene facilitada por la información recibida por el INSS desde el mismo momento en que se expide la baja médica, como por el acceso telemático a toda la información clínica del trabajador, como reiteradamente recoge el RD.

Ciertamente se respeta el derecho del trabajador, pero a través de un acto expreso, lo que evidentemente constituye un condicionante, sobre todo si, como presumimos, queda vinculado a la acción inspectora de los facultativos del INSS o en su caso del servicio público de salud. Actuación de unos y otros inspectores, que tiene como efecto adicional en caso de expedir el alta médica, la asunción de la 
competencia sobre el control futuro de la patología -con la correspondiente perdida de competencias del facultativo que trata al trabajador-, al establecerse que durante los ciento ochenta días naturales siguientes a la fecha en que se expidió el alta, serán estas entidades las únicas competentes, a través de sus propios médicos, para emitir una nueva baja médica por la misma o similar patología.

Finalmente repárese en que es constante en el RD la determinación de reconocer a los inspectores médicos el acceso a la información clínica del trabajador. Determinación que se extiende hasta llegar a la vulneración del artículo 18.1 CE, al establecer en su artículo 8.1 que "en todo caso, los inspectores médicos del Instituto Nacional de la Seguridad Social o, en su caso, del Instituto Social de la Marina, para el ejercicio de sus competencias, tendrán acceso, preferentemente por vía telemática, a la documentación clínica de atención primaria y especializada de los trabajadores del sistema de la Seguridad Social, incluida la documentación clínica de los trabajadores protegidos frente a las contingencias profesionales con las mutuas...".

\section{Conclusión}

El modelo de gestión de la incapacidad temporal se ha transformado en los últimos 20 años, pero las múltiples reformas efectuadas no han encontrado el punto de equilibrio donde se armonicen debidamente los mecanismos de control, los derechos del trabajador, las relaciones con el contrato de trabajo, la participación del empresario. La causa posiblemente esté en una desproporcionada preocupación por el control, no siempre paralela a un desproporcionado gasto.

\section{Bibliografía}

AA.VV.: La incapacidad temporal (Coord. OJEDA AVILES, A.), Tecnos, Madrid, 1996

AA. VV.: Cuestiones en torno a la Incapacidad Temporal (Dir. SEMPERE NAVARRO, A. V.), Cinca, Madrid, 2011;

ALONSO OLEA, M. y TORTUERO PLAZA, J. L., Instituciones de Seguridad Social, Civitas, $18^{\mathrm{a}}$ edic., Madrid, 2006;

ARENAS VIRUEZ, M., "La incapacidad temporal como causa de despido: nulidad o improcedencia”, Relaciones Laborales, núm. 1, 2011;

ARETA MARTÍNEZ, M., "La incapacidad temporal de los funcionarios públicos", AA. VV.: Cuestiones en torno a la incapacidad temporal de los funcionarios públicos, Cinca, Madrid, 2011;

ARRIBAS LÓPEZ, E., "El tan traído y llevado tema de las vacaciones y la incapaci- 
dad temporal en la Administración General del Estado", Actualidad Administrativa, núm. 4, 2014;

AZAGRA SOLANO, M., "Reflexiones sobre el despido del trabajador enfermo", Revista Aranzadi Doctrinal, núm. 4, 2011;

BARBA MORA, A., Incapacidad temporal, Tirant Lo Blanch, Valencia, 2000;

BLASCO LAHOZ, J. F., "La incidencia de la reforma laboral en el sistema público de la Seguridad Social", AA. VV.: Tres años de cambio laboral, Lex Nova, Valladolid, 2013;

BLÁZQUEZ AGUDO, E. Ma., "Presente y futuro de las mutuas de accidentes de trabajo y enfermedad profesional", Aranzadi Social Revista Doctrinal, núm. 2, 2012;

BRUNA REVERTER, J., La incapacidad laboral: problemática legal, jurisprudencial y médica, Comares, Granada, 2012;

CARRIZOSA PRIETO, E., "La tutela del trabajador enfermo en el Estatuto de los Trabajadores", Revista Española de Derecho del Trabajo, núm. 157, 2013;

CASTILLA CASTILLA, Á., "Control médico de la Incapacidad Temporal: la reordenación de las competencias de las entidades que intervienen en la prestación de la Incapacidad Temporal: artículo 128 de la LGSS", Revista de Información Laboral, núm. 3, 2012;

CASTRO CONTE, M., "Evolución de la Seguridad Social de los trabajadores autónomos o por cuenta propia", AA. VV.: Cuestiones en torno a la Incapacidad Temporal (Dir. SEMPERE NAVARRO, A. V.), Cinca, Madrid, 2011;

DUEÑAS HERRERO, L. F., "La contingencia de incapacidad (laboral) temporal en el régimen general de Seguridad Social", Relaciones Laborales, núm. 4, 1996;

FERNÁNDEZ LOMANA, M., "Compatibilidad trabajo-pensión de incapacidad: evolución de la jurisprudencia del Tribunal Supremo", Actualidad Laboral, núm. 2, 2013;

FERNÁNDEZ PRATS, C., La protección de la incapacidad temporal en el régimen general de la Seguridad Social, Tirant lo Blanch, Valencia, 2011;

GALA DURÁN, C., "La compleja relación entre la prestación por incapacidad temporal y el derecho a vacaciones: nuevos criterios judiciales y legales", La administración pública, núm. 10, 2012;

GARCÍA MURCIA J. y ROMÁN VACA, E., "Nuevas pautas de la regulación de la incapacidad temporal", Aranzadi Social, num. 2, 1996;

GARCÍA NINET, J. I., "La incapacidad temporal”, AA.VV.: La incapacidad temporal, (Coord. OJEDA AVILES, A.), Tecnos, Madrid, 1996; 
GARCÍA QUIÑONES, J. C., “Ampliación de la prestación por incapacidad temporal para los trabajadores por cuenta propia”, Documentación Laboral, núm. 70, 2004;

GONZÁLEZ ORTEGA, S., "El control de las bajas médicas como objetivo permanente de las reformas de la incapacidad temporal (I) (El control durante la primera fase de la incapacidad temporal, Relaciones Laborales, núm. 12, 2011; "El control de las bajas médicas como objetivo permanente de las reformas de la incapacidad temporal (y II) (Prórrogas y recaídas. El tránsito hacia la incapacidad permanente), Relaciones Laborales, núm. 13, 2011;

GORELLI HERNÁNDEZ, J., "El derecho al disfrute efectivo de las vacaciones: de la jurisprudencia comunitaria a la regulación estatutaria", Relaciones Laborales, núm. 1, 2014;

JOVER RAMÍREZ, C., La incapacidad temporal para el trabajo. Aspectos laborales y de seguridad social, Tirant lo Blanch, Valencia, 2006;

LÓPEZ INSUA, B. M., La incapacidad temporal en el Sistema de la Seguridad Social, Comares, Granada, 2014; "Vacaciones e Incapacidad Temporal: perspectiva comunitaria y española", Aranzadi Social Revista Doctrinal, núm. 10, 2013;

MARTÍN VALVERDE, A., "Incapacidad laboral transitoria e Invalidez Provisional: legislación y jurisprudencia”, Actualidad Laboral, núm. 39, 1994;

MEDINA MAILHO, R., "Trabajo a tiempo parcial y fijos discontinuos", AA. VV.: Cuestiones en torno a la Incapacidad Temporal (Dir. SEMPERE NAVARRO, A. V.), Cinca, Madrid, 2011;

MUÑOZ MOLINA, J., La incapacidad temporal como contingencia protegida por la Seguridad Social, Aranzadi, Pamplona, 2005;

OLARTE ENCABO, S., "Control y gestión de la incapacidad temporal: análisis crítico del marco jurídico-positivo vigente, el complejo equilibrio entre eficiencia y garantismo", Aranzadi Social Revista Doctrinal, núm. 20, 2011;

PANIZO ROBLES, J. A., "Un nuevo paso en el control de la prestación de la seguridad social por incapacidad temporal: el RD 1430/2009, de 4 de diciembre", Revista de Trabajo y Seguridad Social, CEF, núm. 320, 2009;

PÉREZ ALONSO, M. A., La incapacidad temporal, Tirant lo Blanch, Valencia, 1995;

PURCALLA BONILLA, M. A., "Incapacidad temporal y control del absentismo", AA. VV.: Régimen jurídico y gestión racional del tiempo en la empresa (Dirs. ROMERO BURILLO, A. M y ARGÜELLES BLANCO, A. R.), Aranzadi, Cizur Menor, 2013;

RODRÍGUEZ ÁLVAREZ, V., "Evolución de la normativa sobre la incapacidad para 
el trabajo en el ordenamiento jurídico español", Revista del Ministerio de Trabajo e Inmigración, núm. 94, 2011;

RODRÍGUEZ ESCANCIANO, S., "El control de la incapacidad temporal: su incidencia sobre la contención del gasto público y el aumento de la productividad empresarial", Temas Laborales, núm. 118, 2013;

RODRÍGUEZ-PIÑERO, M., "Derecho a vacaciones y baja del trabajador en la reciente jurisprudencia del TJCE”, Relaciones Laborales, núms. 13/14, 2012;

SALA FRANCO, T., La incapacidad temporal para trabajar derivada de enfermedad o accidente, Tirant Lo Blanch, Valencia, 2005;

SAN MARTÍN MAZZUCONI, C., "El despido del trabajador en situación de incapacidad temporal", AA. VV.: Cuestiones en torno a la Incapacidad Temporal (Dir. SEMPERE NAVARRO, A. V.), Cinca, Madrid, 2011;

TORTUERO PLAZA, J. L., "La incapacidad temporal, contingencia y situaciones protegidas: un análisis teórico", Tribuna Social, núms. 44/45, 1994; "El control público de las Mutuas en materia de incapacidad temporal", AA. VV.: Accidentes de trabajo y Mutuas, La Ley, Madrid, 2008;

TORTUERO PLAZA, J. L. y MORENO ROMERO, F., "Poder de dirección del empresario y los mecanismos de control de la incapacidad temporal: la opción por el control público", AA. VV.: Libertad de empresa y poder de dirección del empresario en las relaciones laborales: estudios ofrecidos al profesor Alfredo Montoya Melgar, Aranzadi, Cizur Menor, 2011;

TORTUERO PLAZA J. L. y SÁNCHEZ-URÁN AZAÑA, Y., "La incapacidad temporal. Régimen jurídico y negociación colectiva", Fundación MAPFRE, Madrid, 1996;

VICENTE PALACIO, M. A., "El control de la Incapacidad Temporal: el control en el ámbito de la relación laboral individual y en el ámbito de la Seguridad Social", Tribuna Social, núm. 168, 2004. 